\title{
Assessing the potential of two customized fiber-optic probes for on-site analysis of bulk feed grains
}

\author{
J.A. Adame-Siles, ${ }^{\text {a* }}$ D. Pérez-Marín, ${ }^{a}$ J.E. Guerrero-Ginel, ${ }^{a}$ A. Larsen ${ }^{b}$ and A. Garrido-Varo ${ }^{a}$ \\ ${ }^{a}$ Agro-livestock Systems Engineering Department, Non-destructive Sensors Unit, University of Córdoba. Campus Rabanales, N-IV, km 396, Córdoba, \\ 14014, Spain. E-mail: *g42adsij@uco.es \\ ${ }^{b}$ Q-Interline, Stengårdsvej 7, DK-4340 Tølløse, Denmark
}

Feed grains are typically transported in bulk and a statistically representative sample of the grain in the truckload is usually required to be taken to the laboratory for wet chemistry or at-line near infrared (NIR) spectroscopy analysis. Currently, most methodologies make use of a physical sampling probe, which mechanically or pneumatically withdraws samples from various depths. Nevertheless, not only is the implementation of this approach expensive and time-consuming, but it is also limited by low sample throughput. In this context, the authors' group is involved in a large research and development project to find more efficient and cost-effective ways of sampling and analyzing bulk raw materials at the reception level. This work presents a piece of this research focused on the evaluation of the optical performance of two fiber-optic probes designed for automated use as immersion probes in truckloads. It is worth noting the rather different optical design of these two diffuse reflectance probes. Probe A features eight bundles ( 37 fibers/bundle), four for measurement and four for illumination, $0.5 \mathrm{~m}$ in length, and four sapphire windows located around the probe diameter. Probe B has one fiber-optic bundle for measurement (7 fibers) and one for illumination (19 fibers), $3 \mathrm{~m}$ in length, and a stainless-steel head with two sapphire windows. The experimental design of this laboratory study aimed at imitating the control of bulk lots of two sort of cereals (maize and wheat). For this purpose, a sample of each cereal was placed into a container ( $0.34 \mathrm{~m}$ in width, $0.4 \mathrm{~m}$ in length and $0.25 \mathrm{in}$ height) for analysis. To avoid interferences caused by design, both probes were attached to the same Fourier transform-NIR instrument (Matrix-F, Bruker Optics), and spectra were acquired in the range 834.2-2502.4nm using the same settings. Two different strategies for recording reference spectra were followed in each case (before the first scan and either after every measurement or after every set of 10 measurements). Noisy regions and spectral repeatability were assessed as a first step towards the evaluation of the feasibility of these probes for performing on-site analysis.

\section{Introduction}

Manufacturing process control, surveillance plans and monitoring programs are key to ensure safety and quality of food and feed materials, as well as to guarantee regulatory enforcement and compliance. ${ }^{1}$ However, this context involves a myriad of challenging tasks to be performed by regulatory bodies, organizations, manufacturers, etc. Thus, there exists an imperative need for protocols and methodologies that can efficiently tackle these issues along the stages of the production chain.
Within this framework, international guidelines and standards proposed by organizations such as ISO, FAO or ISTA have addressed this goal. ${ }^{2}$ Nevertheless, many operational, logistical and methodological problems arise, particularly when bulk raw materials sampling and analysis need to be approached. Despite the fact that there are many critical control points along the agri-food chain, quality control at the reception level is crucial. Not only do the inspections at this point allow the detection of hazards before entering

\section{Correspondence}

J.A. Adame-Siles (g42adsij@uco.es)

doi: $10.1255 /$ nir2017.003

Citation: J.A. Adame-Siles, D. Pérez-Marín, J.E. Guerrero-Ginel, A. Larsen and A. Garrido-Varo, "Assessing the potential of two customized fiber-optic probes for on-site analysis of bulk feed grains", in Proc. $18^{\text {th }}$ Int. Conf. Near Infrared Spectrosc., Ed by S.B. Engelsen, K.M. Sørensen and F. van den Berg. IM Publications Open, Chichester, pp. 3-10 (2019). https://doi. org/10.1255/nir2017.003

\section{(C) 2019 The Authors}

This licence permits you to use, share, copy and redistribute the paper in any medium or any format provided that a full citation to the original paper is given, the use is not for commercial purposes and the paper is not changed in any way.

ISBN: 978-1-906715-27-4 
the process, but they are also vital to preserve quality standards and support traceability in subsequent steps. Moreover, compliance checks of delivery vehicles are of great value in terms of product acceptance, which is key for the industry to validate if suppliers are complying with the conditions of the contract and to detect possible evidences of fraud. This also enables the industry to determine whether to unload a delivery vehicle or not according to its own quality/safety requirements and to implement successful and reliable decision-making systems, by which it would implicitly improve stock and storage management procedures differentiating by quality.

Most existing analytical procedures used in industry concerning quality and safety assessment of raw materials in bulk, such as feed grains, require the taking of samples to the laboratory to be analyzed by time consuming procedures. Current methods use a physical sampling probe, which is driven vertically down into the grain lot and mechanically or pneumatically withdraws samples from various depths. ${ }^{2}$ Then, these samples are often analyzed by classical techniques for the determination of physico-chemical parameters. Although traditional sampling and analysis methods are commonly applied, they include a range of serious shortcomings such as a low or nonexistent degree of lot-sample representativity ${ }^{3}$ (e.g. when sampling is based on single-increment procedures, often referred to as "grab sampling"), the need for qualified manpower, costly implementation or long timelag (depending on the type of analysis required) between sampling and decisions.

Therefore, the implementation of alternative methodologies and procedures addressing the existing constraints of traditional sampling and analysis techniques is imperative. ${ }^{4}$ The availability of advanced technologies to implement on-site analysis is already a reality. In this regard, near infrared (NIR) spectroscopy has become an important source of improvement, contributing to the use of faster, simpler to use and more reliable detecting tools and, hence, to the implementation of more efficient and modern monitoring and control systems. ${ }^{5}$

In addition, this technology has the ability to carry out rapid, low-cost and non-destructive analysis of a variety of types of foods and feeds, including heterogeneous materials. Besides these benefits, recent advances in NIR instrumentation have resulted in the development of time and cost-saving tools to collect spectral information in situ. ${ }^{6,7}$ The possibility of performing on-site qualitative and quantitative analysis has also supported new methodologies combining geospatial and NIR analysis, with which quality attributes are mapped in order to evaluate their spatial distribution in bulk lots of raw materials. ${ }^{8}$ Consequently, the potential of a new evaluation model based on performing sampling and analysis at the same time is of great interest. This would lead to the opportunity of achieving cost-effective and fit-for-purpose protocols with which real-time decisions could be made.

This work aims at performing a preliminary assessment of the optical performance of two custom-built fiberoptic probes for on-site sampling and analysis of raw materials in bulk at the reception level. The specific goals of the evaluation were to study the collection of NIR spectra with both probes and then compare possible spectral differences due to their design.

\section{Materials and methods Instrumentation}

A FT-NIR instrument (Matrix-F, Bruker Optics, Germany) (Figure 1a), supporting multiple modes of analysis and featuring 6 channels to connect fiber optic sensors, was used to perform reflectance analysis. The spectral range available of this equipment is $834.2-2502.4 \mathrm{~nm}$.

Two prototypes of fiber-optic solids probes were interfaced to this instrument to be evaluated. They are characterized by a very different design and form of measurement. On the one hand, one of the probes, henceforth referred to as Probe A (Figure $1 \mathrm{~b}$ ), features a stainless-steel body of $0.5 \mathrm{~m}$ in length and $28.5 \mathrm{~mm}$ in diameter. This probe has four sapphire windows (A, B, C, D) arranged along its longitudinal axis that illuminate and collect light back from the sample. The operating principle is based on two independent pairs of windows, windows $A$ and $C$ work together and so does the pair B-D. Each pair of windows is connected to one channel of the Matrix-F, which means that this probe has a total of four fiber-optic legs. Bundles 1 (illumination) and 2 (collection) are linked to the pair $\mathrm{A}-\mathrm{C}$, whereas bundles 3 (illumination) and 4 (collection) are associated to the pair B-D. Every bundle has a total of 37 fibers, which are $3 \mathrm{~m}$ in length. For this study, a common flat Spectralon diffuse reflectance standard was used to make reference measurements.

Secondly, Probe B (Figure 1c) also has a stainless-steel body but, in this case, of $3 \mathrm{~m}$ in length and $25 \mathrm{~mm}$ in diam- 
(a)

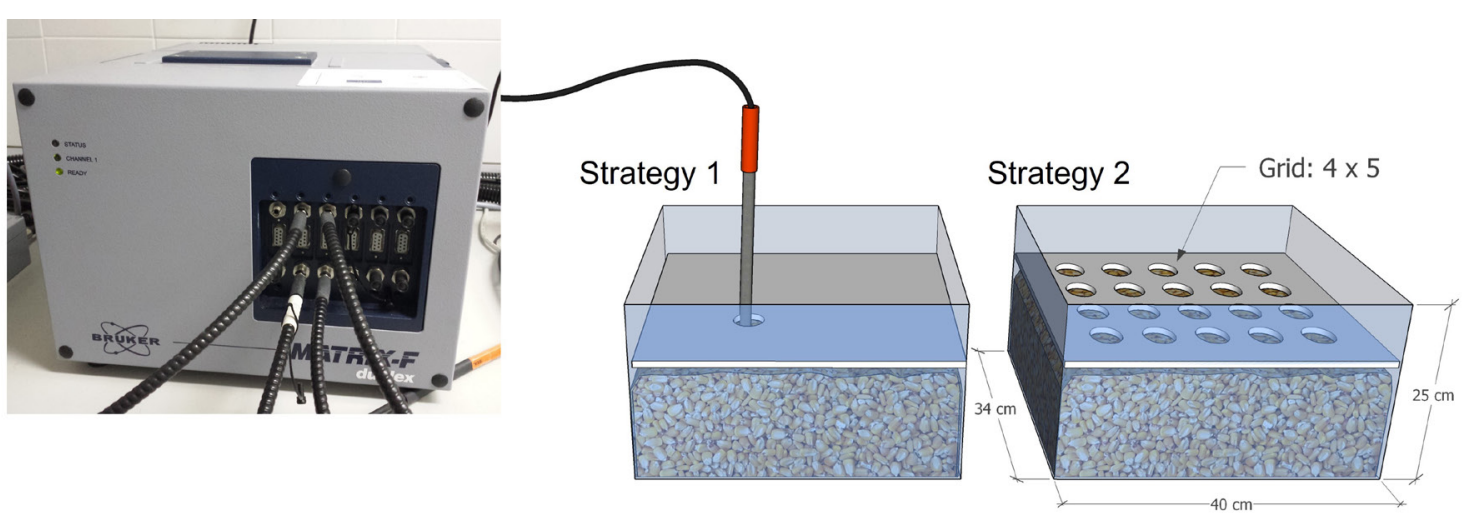

(b)
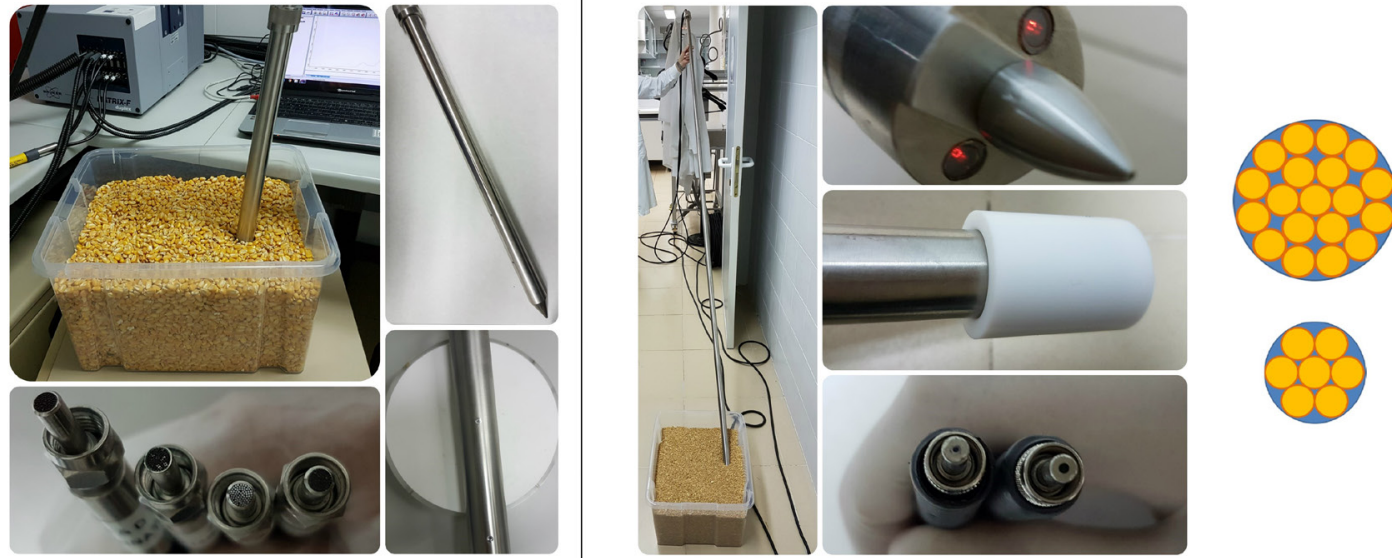

(c)

Figure 1. (a) Instrumental setup with Matrix-F and sampling strategies. (b) Probe A. (c) Probe B.

eter. This probe was connected to the instrument via two fiber-optic cables of $50 \mathrm{~m}$ in length. The collection bundle consists of 7 fibers and the illumination bundle contains a set of 19 fibers (each fiber is $220 \mu \mathrm{m}$ in diameter). The probe head has two sapphire windows at the tip, which are the output for the fiber-optic cables, that irradiate the sample and lead the light back to the spectrometer. In this case, a cylindrical probe-specific Spectralon was used for reference spectra.

\section{Experimental design: samples, sampling and analysis}

Two types of cereal grains were tested in this study, maize and wheat. Samples were placed into a specific plastic container for analysis, where each sample reached a volume of approximately $20.4 \mathrm{dm}^{3}$.

Two sampling strategies were carried out to evaluate the optical performance of both probes with the two kinds of cereals grains. First, Strategy 1 consisted of only one sampling point, while Strategy 2 was based on a grid of $4 \times 5$ sampling locations.

NIR spectroscopy analysis with both probes was performed following two different sequences in the case of Strategy 1. In Sequence 1, sample was measured right after reference was taken, and this was repeated 20 times. For Sequence 2, however, reference was taken only once, just before the analysis of the sample (repeated 10 times, without removing the probe from its position). In contrast, Strategy 2 was carried out applying one sequence, which included the measurement of the reference and then the analysis of the grid of 20 sampling points.

The settings for the spectral acquisition were the same in all cases (32 scans and a resolution of $16 \mathrm{~cm}^{-1}$ ). After the set of tests was performed, the noise level along the spectral range available from the spectrometer was evaluated. For this purpose, a first derivative pre-treatment 
with a single-unit gap and five data-point smoothing was applied to the spectral data. The Root Mean Square (RMS) statistic was used for the study of similarity between spectra corresponding to replicates of the same sample, and was calculated by using the following equation:

$$
R M S_{j}=\sqrt{\frac{\sum_{i=1}^{n} D_{i j}^{2}}{n}} ; \quad D_{i j}=y_{i j}-\bar{y}_{i}
$$

where $n$ is the number of wavelengths, $y_{i j}$ is the $\log (1 / R)$ for the sub-sample $j$ at $\lambda_{i}$ and $\bar{y}_{i}$ is the $\log (1 / R)$ for the averaged spectrum of a sample at $\lambda_{i}$. MEAN and STD parameters were also calculated by:

$$
\text { MEAN }=\sqrt{\frac{\sum_{j=1}^{n}\left(R M S_{j}\right)^{2}}{N}} ; S T D=\sqrt{\frac{\sum_{j=1}^{n}\left(R M S_{j}\right)^{2}}{N-1}}
$$

where $N$ is the number of sub-samples.

\section{Results and discussion}

First, noisy regions were assessed so as to determine the total useful wavelength range in each case, as well as to standardize the spectral responses before carrying out the following steps of the study. Regions of noise were found at both ends of the spectrum with Probe $A$ and Probe B. Spectra were transformed using a first-order derivative (single-unit gap and five data-point smoothing) to detect the spectral regions of random oscillations in absorbance values. After visual examination of the noisy areas, the optimum working range selected was 1120-2040 nm, which was the one obtained by Probe $\mathrm{B}$ which was more restrictive than by Probe $\mathrm{A}$. This can be attributable to the fact that the length of the fibers to connect Probe B to the Matrix-F was $50 \mathrm{~m}$, which was much higher than the length used by Probe $A(3 \mathrm{~m})$. It has been studied that there exists a direct relationship between the length of the fiber-optic cables and the level of noise, as the light power propagating in a fiber decays exponentially with length due to absorption and scattering losses.

Results of the analysis following the sampling Strategy 1 are shown in Figure 2. It can be noted that Sequence 1 , which consisted of measuring the reference-sample 20 times, shows a much higher dissimilarity in the level of the absorbance values between replicates than Sequence 2, in which reference was taken once and then sample 10 times without moving the probe from its position. It is a reasonable result if the physical nature of both products being analyzed is considered, besides the tools and type of the analysis performed in each case. On the one hand, it should be highlighted the rather different designs not only of the probes, but also of the references. Concerning the latter, while Probe B had a custom-built one available, adapted to efficiently collect reference spectra with this probe, Probe A did not have any specific-probe reference during the development of this study, so a common flat Spectralon had to be used here. Therefore, the spectral differences found along the $y$-axis can be partly seen as a consequence of this, these differences are accentuated in Sequence 1 due to the need to take reference measurement at each iteration. Factors such as size, shape, brightness, orientation and density of the material may have also played an important role to explain some of the differences observed between products. ${ }^{10,11}$ Maize has generally shown less spectral repeatability than wheat in this study. Among other aspects, this behavior might be originated from the fact that maize particles are larger than wheat grains, which may have made more difficult for the probes to analyze the sample, as a greater interparticle space increases the presence of air inside.

Figure 3 compares MEAN and STD statistics of Probe A versus Probe B for each sequence and cereal. Results confirm the initial assessment from the visual examination of the spectra. Sequence 1 shows a much lower spectral repeatability than Sequence 2. Considering Sequence 1, Probe $\mathrm{A}[0.38 \log (1 / R)]$ demonstrates a higher variation than Probe B $[0.12 \log (1 / R)]$, both in maize and wheat grains. Sequence 2 shows how Probe B statistics are slightly higher for maize than Probe A values, which is even more apparent for wheat $[7200 \mu \log (1 / R)$ versus $2000 \mu \log (1 / R)$, respectively]. This suggests that Probe A reference has played a relevant role in the results as it could be anticipated above.

On the other hand, the results for the Strategy 2, based on a grid of 20 sampling points, are shown in Figure 4 . In this case, Probe A and Probe B spectra significantly differ in their quality. Probe A achieves to represent typical peaks both for maize and wheat. However, random oscillations can be seen in the selected wavelength range for the spectra taken by Probe B.

Figure 5 compares once again MEAN and STD statistics of both probes. Probe A reveals to present a higher variation than Probe $B$ in this case. Despite the apparent differences in repeatability, the oscil- 


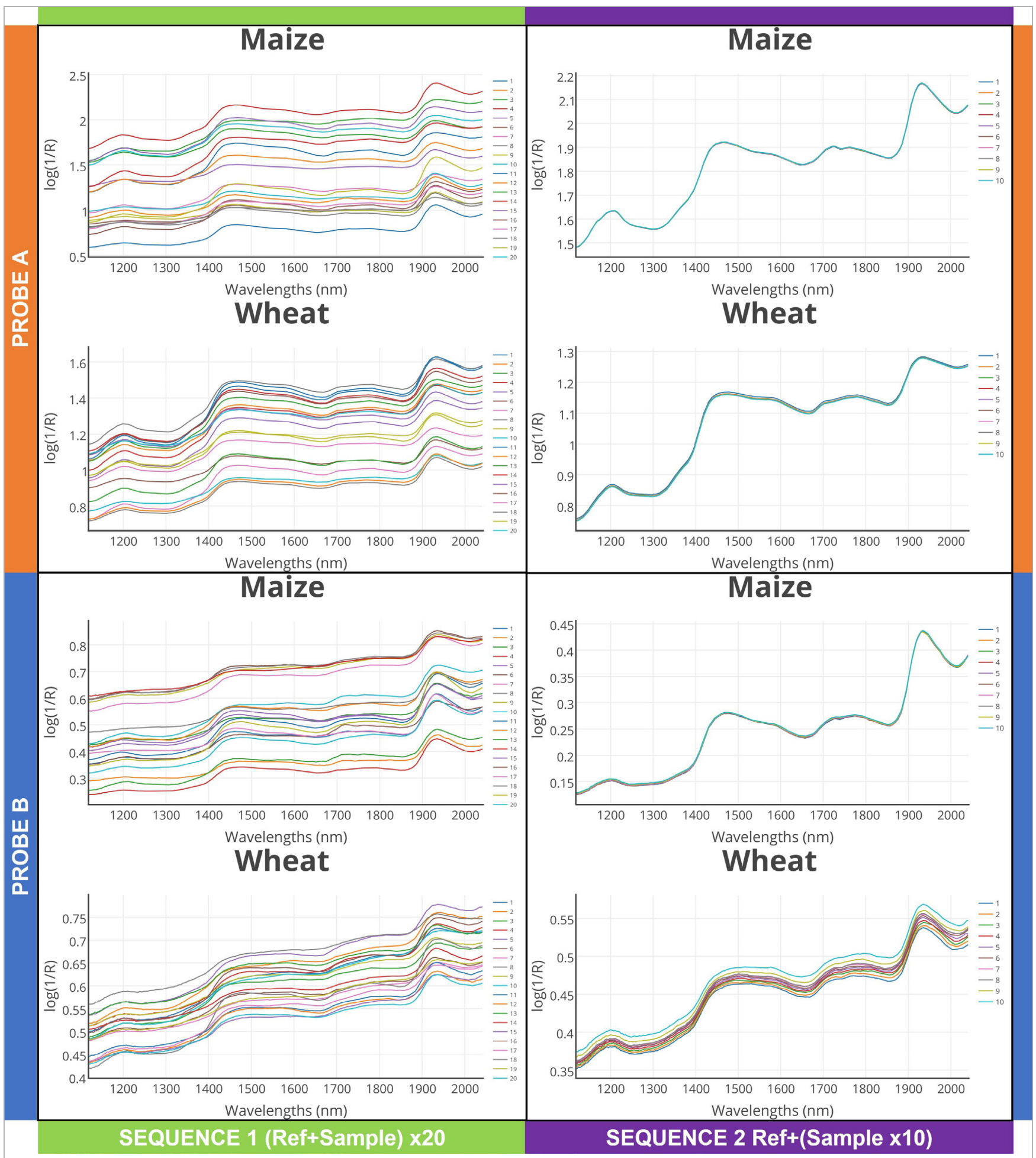

Figure 2. Resulting NIR spectra from Strategy 1.

lations observed in Probe B spectra suggest that it would hardly be possible to infer valuable information in this case. For this reason, more work will be needed regarding the improvement of the ratio signal/noise especially with this probe.

\section{Conclusions}

The adoption of on-site analysis of foods and feeds is of great value for safety/quality assurance purposes. Rapid and cost-effective assessment of raw materials is required 

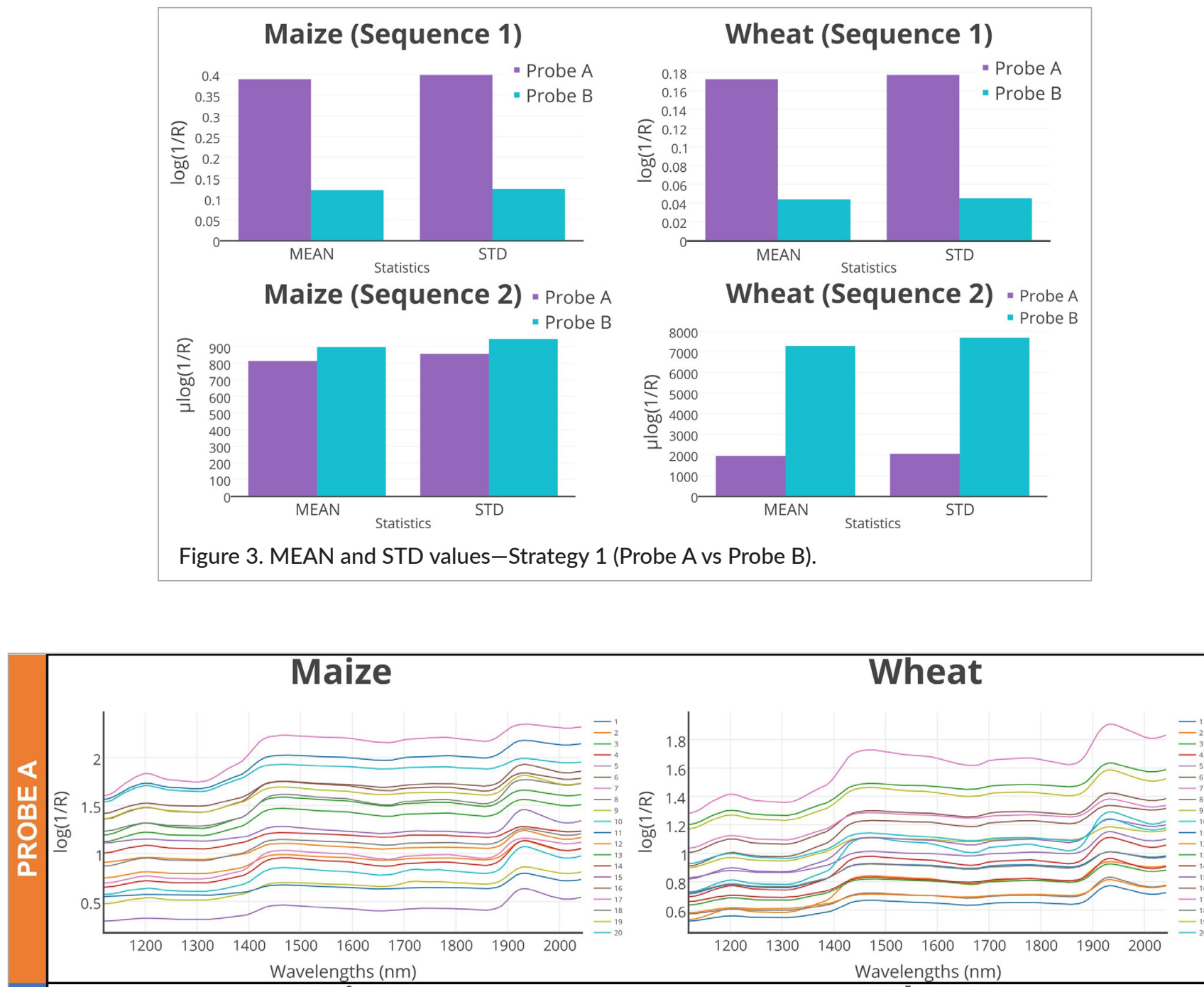

Maize

Wheat
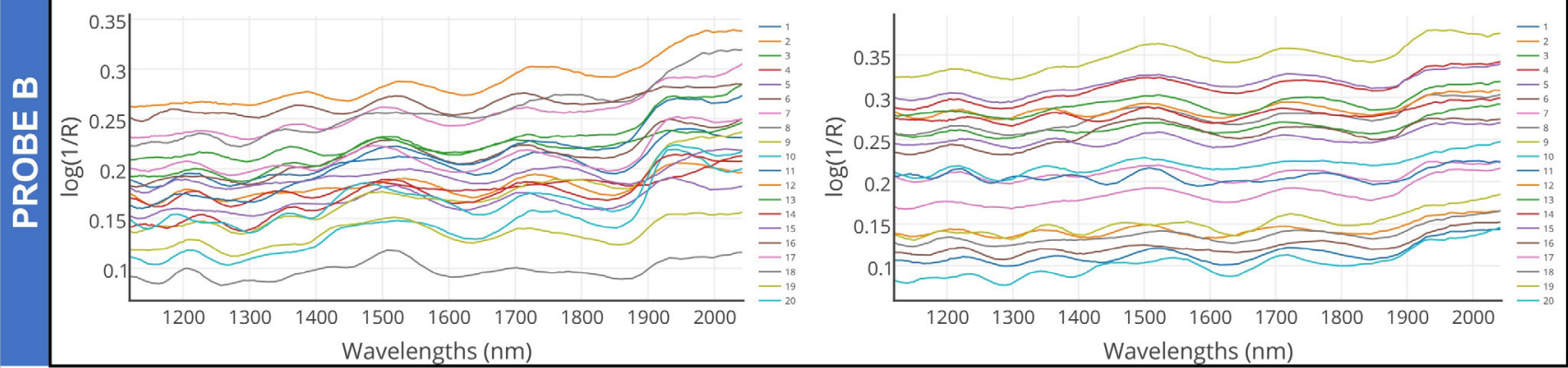

SEQUENCE Ref+(Sample X20)

Figure 4. Resulting NIR spectra from Strategy 2.

within the context of process control and surveillance protocols. The development and application of fast and nondestructive analytical methods and techniques is of particular interest in order to efficiently evaluate bulk products right at their entrance to the processing plant. This study explores the possibility of applying innova- tive technologies such as NIR spectroscopy to sample and analyze large volumes of raw materials. Towards this end, two different insertion probe systems for spectral analysis were designed. The present work approaches a preliminary comparison of the spectral performance of both fiber-optic probes. Results indicate that Probe 

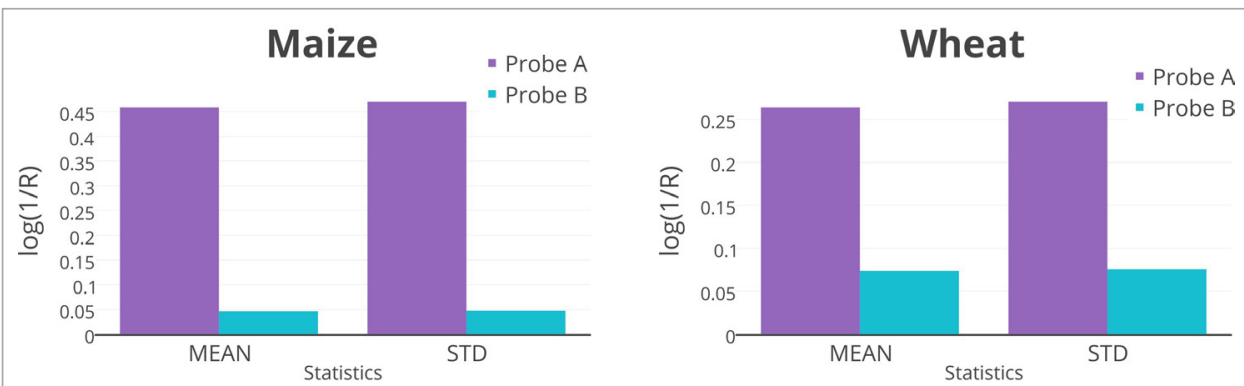

Figure 5. MEAN and STD values-Strategy 2 (Probe A vs Probe B).

A comparatively achieved a lower spectral repeatability than Probe B. Notwithstanding, visual examination of the spectra for every strategy and sequence showed that valuable spectral information is more likely to be found in Probe A spectra. Most spectral differences encountered between replicates in Probe A spectra could be attributable, to a large extent, to the use of an inappropriate and non-specific reference in this case. In fact, Probe A achieves better results than Probe B when reference measurements are less involved (Strategy 1 , sequence 2). Furthermore, its design shows potential to explore greater areas than Probe B, as it can perform measurements at different depths at the same time, and to achieve a better optical performance, as it is based on bundles with a greater fiber-optic density. Thus, on-going research is focused on the development of a probe-specific reference for Probe A, in order to carry out feasibility tests which may lead to its potential application in real conditions.

\section{References}

1. European Commission, "Regulation (EC) No. $882 / 2004$ of the European Parliament and of the Council of 29 April 2004 on Official Controls Performed to Ensure the Verification of Compliance with Feed and Food Law, Animal Health and Animal Welfare Rules", Off. J. Eur. Union L 165(April), 1-141 (2004).

2. International Organization for Standardization (ISO), ISO 24333:2009 - Cereals and cereal products -Sampling (2009).

3. K.H. Esbensen, DS 3077. Representative Sampling Horizontal Standard. Danish Standards (2013).

4. F. Cheli, A. Campagnoli, L. Pinotti and V.D. Orto, Rapid Methods as Analytical Tools for Food and Feed
Contaminant Evaluation: Methodological Implications for Mycotoxin Analysis in Cereals, Food Production: Approaches, Challenges, and Tasks. Intech Open (2012). http://www.intechopen.com/books/foodproduction-approaches-challenges-and-tasks [accessed Oct 16 2017].

5. E. Fernández-Ahumada, A. Garrido-Varo, J.E. Guerrero-Ginel, D. Pérez-Marín and T. Fearn, "Taking NIR calibrations of feed compounds from the laboratory to the process: Calibration transfer between predispersive and postdispersive instruments", J. Agric. Food Chem. 56, 10135-10141 (2008). doi: https://doi.org/10.1021/jf801881n

6. E. Zamora-Rojas, D. Pérez-Marín, E. de Pedro-Sanz, J.E. Guerrero-Ginel and A. Garrido-Varo, "Handheld NIRS analysis for routine meat quality control: database transfer from at-line instruments", Chemometr. Intell. Lab. Syst. 114, 30-35 (2012). doi: https://doi. org/10.1016/j.chemolab.2012.02.001

7. A. Garrido-Varo, S. Vega, F. Maroto-Molina, M. José de La Haba and D. Pérez-Marín, "On-site quality control of processed land animal proteins using a portable micro-electro-mechanical-systems near infrared spectrometer", J. Near Infrared Spectrosc. 24(1), 47 (2016). doi: https://doi.org/10.1255/ jnirs.1192

8. J.A. Adame-Siles, T. Fearn, J.E. Guerrero-Ginel, A. Garrido-Varo, F. Maroto-Molina and D. PérezMarín, "Near-infrared spectroscopy and geostatistical analysis for modeling spatial distribution of analytical constituents in bulk animal by-product protein meals", Appl. Spectrosc. 71(3), 520-532 (2017). doi: https://doi.org/10.1177/0003702816683958

9. A. Sanchez-Bonilla, A. Garrido-Varo, F. MarotoMolina, J.E. Guerrero-Ginel and B. de La RozaDelgado, "Influence of the fiber optic length on the repeatability of the near infrared spectroscopy 
signal", in Proceedings of the 17th International Conference on Near Infrared Spectroscopy. Foz do Iguassu, Brazil (2015).

10.P.C. Williams. "Application of near infrared reflectance spectroscopy to analysis of cereal grains and oil seeds", Cereal Chem. 52, 516-576 (1975).

11. L.A. Mohan, C. Karunakaran, D.S. Jayas and N.D.G. White, "Classification of bulk cereals using visible and NIR reflectance characteristics", Canadian Biosyst. Eng. 47(7), 7-14 (2005). 